\title{
Narraciones escritas en niños con Trastorno Específico del Lenguaje (TEL)
}

\section{Written Narration in Specific Language Impairment (SLI)}

Recibido: octubre 6 de 2010 | Revisado: octubre 11 de 2011 | Aceptado: junio 10 de 2012

\author{
MANUEl SORIANO-FERRER * \\ Universidad de Valencia, España \\ Ma CARMEN CONTRERAS-GONZÁlEZ ** \\ Equipos de Orientación Educativa, Junta de Andalucía \\ (Almería), España
}

SICI: 2011-2277(201212)11:4<1341:CENTEL>2.0.CO;2-M

Para citar este artículo: Soriano-Ferrer, M. \& Contreras-González, M. C. (2012) Narraciones escritas en niños con Trastorno Específico del Lenguaje (TEL). Universitas Psychologica, 11(4), 1341-1351.

* Profesor Titular de Universidad en el Departamento de Psicología Evolutiva y de la Educación. E-mail: Manuel.Soriano@uv.es

** Doctora en Psicología por la Universidad de Granada y actualmente psicopedagoga de los Equipos de Orientación de la Junta de Andalucía. Miembro del Grupo de Investigación CTS-135 "Neuropsicología de la Atención" del Departamento de Neurociencia y Ciencias de la Salud de la Universidad de Almería. E-mail:mccontre@ual.es

\section{RES UMEN}

El presente estudio examinó las narraciones escritas, así como la influencia de las variables lingüísticas sobre la competencia narrativa de los niños con Trastorno Específico del Lenguaje (TEL). Veinticinco niños con TEL y 25 niños con desarrollo normal del lenguaje, equilibrados en edad e inteligencia, realizaron una tarea de narración escrita. Los resultados indican que ambos grupos difieren en la producción de narraciones. Los niños con TEL no solo escribieron historias más cortas, sino también de menor organización y coherencia. Además cometieron significativamente más errores de ortografía natural y de sintaxis. Los análisis de regresión jerárquica mostraron que diferentes medidas lingüísticas - memoria secuencial auditiva, comprensión auditiva y expresión verbal- tienen un peso predictivo de la competencia narrativa.

Palabras clave autores

Trastorno Específico del Lenguaje, habilidades narrativas, gramática de la historia. Palabras clave descriptores

Trastorno Específico del Lenguaje, narración escrita, lenguaje.

\section{A B S T R A C T}

The present study examined the written narrations and the influence of linguistic measures on narrative competence of children with specific language impairment (SLI). A narration task was used to assess narrative abilities in 25 children with SLI and 25 normally developing children, matched on age and IQ. Results indicated that the two groups did differ in the production of narratives. The children with SLI provided short narratives, poorly organized and less cohesive. Also, writings were characterized by more syntax errors and had high proportion of phonologically inaccurate spelling errors. Hierarchical regression analysis showed that different linguistic measures - auditory comprehension, verbal fluency and sequential auditory memory - played a predictive role in narrative competence.

Key words authors:

Specific Language Impairment, Narrative Abilities, Story Grammar.

Key words plus:

Specific Language Impairment, Written Narration, Language. 


\section{Introducción}

Los niños con Trastorno Específico del Lenguaje (TEL) experimentan problemas en la adquisición y el procesamiento del lenguaje oral, que no son explicados por déficits cognitivos, neurológicos o perceptivos (Bishop \& Leonard, 2001; Mendoza, 2001). Se estima que el TEL tiene una prevalencia aproximada del 7\% de los niños (Tomblin et al., 1997), siendo más frecuente en varones que en mujeres (Choudhury \& Benasich, 2003).

Las dificultades lingüísticas de estos niños son muy heterogéneas, variando en severidad, persistencia y en cuanto a los planos del lenguaje afectados, tales como la fonología, el léxico y la semántica, sintaxis, morfología y pragmática (Aguado, 1999; Bishop \& Leonard, 2001; Chevrie-Muller, 1997; Hincapié-Henao et al., 2008; Mendoza, 2001; Monfort \& Juárez, 1993; Restrepo \& Gutiérrez-Clellen, 2001; Sanz, 2002). Además, estas deficiencias del lenguaje oral persisten entre el 50 y el $60 \%$ de los chicos con TEL en la adolescencia y en la edad adulta (p.e., vocabulario más reducido, problemas con la morfología derivativa y flexiva, y con algunas estructuras sintácticas), destacándose así su carácter crónico (Bishop \& Leonard, 2001; Clegg, Hollis, Mawhood \& Rutter, 2005; Howlin \& Udwin, 2002; Norbury, Bishop \& Briscoe, 2001).

Desafortunadamente, dichas dificultades lingüísticas afectan negativamente su capacidad para el discurso narrativo oral que tiene importantes repercusiones sobre el funcionamiento social, lingüístico y académico del niño, porque exige la capacidad de tener en cuenta las necesidades informativas del oyente y utilizar formas lingüísticas con finalidades comunicativas (Leinonen, Letts \& Smith, 2000). Mediante el empleo de diferentes metodologías de elicitación (p. j., dibujos, filmaciones, conversaciones, repetición, etc.), numerosos estudios que han analizado las habilidades de narración oral (e.g. Befi-Lopes, Bento \& Perissinoto, 2008; Botting, 2002; Contreras \& Soriano, 2004; Copmann \& Griffith, 1994; Epstein \& Phillips, 2009; Liles, Duffy, Merrit \& Purcell, 1995; Manhardt \& Rescorla, 2002; Merrit \& Liles, 1987) han encontrado que los niños con TEL presentan dificultades en diferentes aspectos de la narración: a) introducen en la historia menos componentes de la gramática, por lo que sus relatos son más cortas, con menos ideas, tanto principales como secundarias; sin embargo, en ocasiones, pueden llegar a añadir información no pertinente para producir una narración de una longitud razonable; b) en el proceso de narración, que requiere también la traducción de ideas en palabras, muestran mayores dificultades de acceso al léxico que los sujetos de los grupos de comparación ; c) exhiben menor uso de mecanismos de cohesión lingüística, lo cual dificulta la comprensión del oyente al tener que inferirlos y d) registran dificultades para secuenciar la historia y respetar el orden temporal en el que se presentan los acontecimientos. En definitiva, los chicos con TEL presentan dificultades a la hora de establecer los elementos que organizan la narración: la coherencia estructural de la historia y la cohesión lingüística.

Por otro lado, dado que el lenguaje escrito se asienta sobre el lenguaje oral, existen razones para predecir que las deficiencias lingüísticas de los chicos con TEL también podrían tener un impacto negativo sobre el lenguaje escrito en general y, sobre la narración escrita en particular, ya que la escritura incrementa las exigencias de organización y planificación de la información. De hecho, Gillam y Johnston (1992) confirmaron que para estos chicos era mucho más complicado escribir una historia que narrarla de forma oral. Por ello, el lenguaje escrito ha sido definido como una ventana a los déficits lingüísticos residuales de los niños (Bishop \& Clarkson, 2003).

Los diferentes aspectos por medio de los cuales es posible predecir dificultades en el lenguaje escrito, se pueden agrupar en tres dominios: lenguaje, lectoescritura y memoria de trabajo. Así, las dificultades que tienen los niños con TEL en el acceso léxico y en la construcción de frases, podrían conducirles a escribir textos más cortos, con menos vocabulario, con sintaxis más simple y con errores sintácticos. Sus problemas con la conciencia fonológica producirían un aumento de errores de ortografía natural en sus escritos. Por otro lado, numerosos estudios han señalado el rol decisivo de la memoria de trabajo en la comprensión del lengua- 
je y de la lectura (Montgomery, 2003). Por esto, la textualización de las ideas en escritura incrementa las exigencias cognitivas, por lo que estos chicos podrían saturar la memoria de trabajo al escribir. Por otro lado, la adquisición de la lectoescritura podría tener un rol moderador de las dificultades de los chicos con TEL, ayudándoles a compensar sus dificultades con el lenguaje oral. Sin embargo, a pesar de la gran cantidad de evidencias indirectas de dificultades, son escasos los trabajos que han analizado el lenguaje escrito de los chicos con TEL. Los estudios desarrollados hasta el momento han señalado que sus escritos se caracterizan por: a) mayor cantidad de errores gramaticales, especialmente con los verbos compuestos (Gillam \& Johnston, 1992; Scott \& Windsor, 2000); b) mayor cantidad de errores ortográficos (Bishop \& Clarkson, 2003), especialmente errores de ortografía natural (Mackie \& Dockrell, 2004) y c) menor riqueza de vocabulario (Bishop \& Clarkson, 2003). Las mismas limitaciones apuntadas por los estudios previos también se han encontrado en chicos con TEL de mayor edad (Dockrell, Lindsay \& Connelly, 2009; Dockrell, Lindsay, Conelly \& Mackie, 2007). Estos dos últimos estudios han mostrado también que sus escritos eran más cortos, tenían una mayor cantidad de errores sintácticos, así como dificultades con la organización de las ideas. Pero, sin ninguna duda, el resultado más novedoso de estos estudios ha sido la confirmación empírica del poder explicativo que diferentes habilidades de lenguaje oral (vocabulario, morfosintaxis, comprensión, etc.) tenían sobre el lenguaje escrito.

Desde estas consideraciones y dada la carencia de este tipo de trabajos en nuestra lengua, el presente trabajo se propuso examinar las diferencias en las habilidades de narración en español de niños en edad escolar con TEL y niños con desarrollo normal del lenguaje. Concretamente, en este estudio se han planteado los tres objetivos siguientes: 1) analizar si los niños con TEL muestran una sensibilidad a las características estructurales de la historia, diferente a la de los niños con desarrollo normal del lenguaje; 2) analizar si los errores en las narraciones de los niños con TEL difieren de los errores que cometen los niños con desarrollo normal del lenguaje y 3 ) determinar el poder predictivo de diferentes habilidades psicolingüísticas -comprensión auditiva, memoria secuencial auditiva, expresión verbal e integración gramatical- en las narraciones escritas. Para ello, se utilizó una tarea de narración escrita de un cuento previamente oído por los niños. Además, en el análisis de las narraciones se han conjugado distintas aproximaciones tradicionales en los estudios previos como las categorías de la gramática de la historia y los análisis de los errores en el recuerdo escrito de la historia, junto con medidas novedosas de integridad de la misma (Lê, Coelho, Mozeiko \& Grafman, 2011).

\section{Método}

\section{Sujetos}

Participó en este estudio, un total de 50 niños de clase social media baja, de edades comprendidas entre los 7 y los 9 años $(M=8.3$ años y $D E=0.55)$, escolarizados en colegios públicos de la provincia de Almería, de los cuales 36 eran niños y 14 niñas, lo que representa un $72 \%$ y un $28 \%$, respectivamente. De la muestra total, 25 niños presentaban un Trastorno Específico del Lenguaje (TEL), mientras que los 25 restantes constituyeron el grupo de comparación normal. A su vez, de los 25 niños que formaban el grupo con TEL, 17 (68\%) eran niños y 8 (32\%) eran niñas, y de los 25 niños que formaban el grupo de comparación normal, 19 (76\%) eran niños y 6 (24\%) eran niñas.

Los criterios de selección de los 25 niños con TEL estuvieron basados en los siguientes criterios diagnósticos (Aguado, 1999; American Psychiatric Association [APA], 2000; Bishop \& Leonard, 2001; Castro-Rebolledo, Giraldo-Prieto, Hincapié-Henao, Lopera \& Pineda, 2004; Chevrie-Muller, 1997; Hulme \& Snowling, 2009; Mendoza, 2001; Monfort \& Juárez, 1993; Stark \& Tallal, 1981): a) ausencia de problemas auditivos y de historias de otitis; b) ausencia de anomalías en la estructura oral y en la función motora oral; c) tener un CI no verbal normal $\left(\mathrm{Pc}_{\mathrm{c}} \geq 50\right)$ en el Test de Matrices Progresivas (Raven, 1995); d) ausencia de problemas neurológicos y psicopatológicos; e) presentar 
un ajuste socioemocional adecuado a su edad y f) presentar un retraso de dos o más años en edad psicolingüística en el Test Illinois de Habilidades Psicolingüísitcas [ITPA, por sus siglas en inglés] (Kirk, McCarthy \& Kirk, 1986). Estos niños no presentaban dificultades articulatorias, aunque algunos de ellos las habían mostrado en el pasado, pero las habían superado al final de la etapa de educación infantil.

Para la obtención de la muestra del grupo de comparación normal (25 sujetos), se solicitó la colaboración de los Equipos de Orientación Escolar (EOEs). Los criterios que se adoptaron para la selección fueron: a) rendimiento académico normal; b) una puntuación de CI no verbal normal $(\mathrm{Pc}>50)$ en el Test de Matrices Progresivas (Raven, 1995) y c) ausencia de problemas del lenguaje o del aprendizaje, psicosis o daño neurológico, déficits sensoriales o motores.

La comparación de los niños con TEL y los niños del grupo de comparación señala que no difieren en cuanto a la edad cronológica, $U_{(48)}=229$, $p<0.105, d=-0.57$, ni a la inteligencia no verbal (Raven), $U_{(48)}=275, p<0.359, d=-0.26$. Sin embargo, como era de esperar, se encontraron diferencias significativas en edad psicolingüística (ITPA), $F_{(1,48)}=244.47, p<0.000, d=-5.08$.

\section{Instrumentos de evaluación y procedimiento}

Con el fin de evaluar la capacidad narrativa de los niños se empleó una tarea de narración escrita del cuento "El conejo y el puma" (Borzone \& Diuk, 2003). Se trata de un relato compuesto por 19 proposiciones y tiene una estructura simple, que sigue las categorías de la gramática de la historia de Stein y Glenn (1979).

Todos los niños fueron evaluados individualmente por los EOEs. El evaluador daba a los niños las siguientes instrucciones: "Ahora te voy a leer dos veces un cuento. Debes estar muy atento porque luego tienes que escribirlo". Los escritos de los niños fueron valorados posteriormente en función de dos bloques de medidas: medidas de gramática de la historia y errores de producción.

Las medidas de gramática de la historia tomadas fueron: 1) proporción del total de proposiciones recordadas, que se calculó contabilizando el número total de proposiciones que respetaban el contenido semántico de la proposición, no necesariamente su literalidad, dividiéndolo por el total de proposiciones del cuento y multiplicando el resultado por $100 ; 2$ ) proporción de proposiciones por categorías o número de proposiciones recordadas de cada una de las categorías de Stein y Glenn (1979), esto es, introducción, suceso, respuesta interna, ejecución, consecuencia directa y resolución, dividido por el número total de proposiciones de cada una de ellas y multiplicando el resultado por 100; 3) Integridad de la historia, calculada por contabilización del número total de las proposiciones fundamentales de la historia, dividiéndolo por ocho - que eran los elementos fundamentales de la misma-, y multiplicando el resultado por 100 , tal como han propuesto

TABLA 1

Comparación de los sujetos con TEL y sujetos con desarrollo normal en variables relacionadas con la habilidad narrativa

\begin{tabular}{|c|c|c|c|c|c|c|c|}
\hline & \multicolumn{2}{|c|}{$\begin{array}{c}\text { Grupo Trastorno } \\
\text { Específico de Lenguaje } \\
(N=25) \\
\end{array}$} & \multicolumn{2}{|c|}{$\begin{array}{l}\text { Grupo de comparación } \\
\qquad(N=25)\end{array}$} & \multirow[b]{2}{*}{$\begin{array}{c}\text { Estadístico } \\
F / U\end{array}$} & \multirow[b]{2}{*}{$\begin{array}{l}\text { Signif. } \\
\text { Bilat. }\end{array}$} & \multirow[b]{2}{*}{$\begin{array}{c}d \text { de } \\
\text { Cohen }\end{array}$} \\
\hline & $\begin{array}{c}\text { Media } \\
(D E)\end{array}$ & $\begin{array}{l}\text { Mediana } \\
\text { (IQR) }\end{array}$ & $\begin{array}{l}\text { Media } \\
(D E)\end{array}$ & $\begin{array}{l}\text { Mediana } \\
(\mathrm{IQR})\end{array}$ & & & \\
\hline Edad & $8.2(0.66)$ & $8.11(0.55)$ & $8.5(0.38)$ & $8.6(0.75)$ & $U=229$ & 0.105 & -0.57 \\
\hline Edad Psicolingüística (ITPA) & $6.09(0.64)$ & - & $8.71(0.53)$ & - & $F=244.47$ & 0.000 & -5.08 \\
\hline CI no verbal (Raven) & $59(12.24)$ & $50(25.00)$ & $56(10.9)$ & $50(12.50)$ & $U=275.00$ & 0.359 & 0.26 \\
\hline
\end{tabular}

Nota. Los datos se presentan como media/desviación típica, en el caso de seguir una distribución normal y/o como mediana/rango intercuartílico (IQR) en caso contrario.

Fuente: elaboración propia. 
Lê et al. (2011). Se consideraron como elementos fundamentales las ocho proposiciones pertenecientes a distintas categorías de la historia, seleccionadas por siete jueces como esenciales para reflejar la cadena de acontecimientos especio-temporales fundamentales de la historia. Aquellas proposiciones que no fueron seleccionadas por todos los jueces no se consideraron como fundamentales (Lê et al., 2011).

Por último, se codificaron tres errores de producción en las narraciones que son habituales en la evaluación del discurso escrito (Cervera \& Toro, 1984): a) Ortografía natural. Se contabilizaron todos las fallas que transforman la fonética de la palabra como los errores de sustituciones, rotaciones, omisiones, adiciones, inversiones, uniones y fragmentaciones indebidas; b) Ortografía arbitraria/ reglada. Se contabilizaron los errores de acentuación, signos de puntuación, cambios consonánticos (p. e., v/b, j/g, ll/y, x/ch, r/rr, h) así como la inobservancia de las reglas ortográficas; c) Errores sintácticos en el uso del número, género, tiempos verbales, orden de las palabras en las oraciones, omisiones, sustituciones o adiciones de palabras función (p. e., artículos, preposiciones, adverbios), la incoherencia en el texto, así como la enumeración de palabras sin concordancia sintáctica o la enumeración perseverativa de frases.

La evaluación de las narraciones fue realizada por tres licenciadas en Psicología previamente entrenadas, obteniéndose una concordancia interjueces alta (kappa de Cohen), que osciló entre 0.87 y 1 en las diferentes variables empleadas en el análisis (Landis \& Koch, 1977).

\section{Análisis de datos}

Para nuestros dos primeros objetivos, dirigidos a comparar las posibles diferencias entre el grupo de comparación y el grupo TEL, se comprobó que los datos cumplían el criterio de normalidad estadística, aplicando la prueba de KolmogorovSmirnov. En aquellos casos en que la distribución era normal $(p>0.05)$ se realizaron análisis de varianza (ANOVA) de comparación entre grupos, mientras que el test $U$ de Mann-Whitney fue empleado en caso contrario. Se definió como significativo el valor $p$ bilateral inferior a 0.05. Además, se aportó el tamaño del efecto usando la d de Cohen (Cohen, 1988), cuyo valor sería negativo cuando los niños con TEL puntuaran más bajo que el grupo de comparación, en las diferentes variables.

Para la consecución de nuestro tercer objetivo se llevó a cabo un análisis de regresión múltiple, con el fin de determinar la capacidad de predicción de cuatro variables psicolingüísticas sobre la integridad de la historia. Las variables psicolingüísticas del ITPA que se introdujeron como predictoras, fueron la comprensión auditiva, la memoria secuencial auditiva, la expresión verbal y la integración gramatical. Como variable dependiente se empleó la integridad de la historia. El método de inclusión de variables fue el de paso a paso, con criterios de inclusión $p<0.05$ y de exclusión $p>0.1$. Se optó por este criterio de selección estadística debido al tamaño de la muestra.

\section{Resultados}

\section{Resultados en medidas de gramática de la historia}

Los resultados que aparecen en la Tabla 2 indican que los niños con TEL y los normales difieren en el número total de proposiciones recordadas, $U_{(48)}=34, p<0.000, d=-2.19$, siendo el grupo de comparación el que emplea mayor número de proposiciones. En otras palabras, el número total de proposiciones de los niños con TEL se encuentra a más de dos desviaciones típicas por debajo con respecto a las proposiciones recordadas por escrito por el grupo de comparación.

En cuanto al análisis realizado sobre el recuerdo escrito de las diferentes categorías contempladas en la gramática de la historia, los niños con TEL recuerdan un menor número de proposiciones en las diferentes categorías, situándose a una o casi dos desviaciones típicas por debajo del grupo de comparación. Aparecen diferencias significativas en la proporción de proposiciones recordadas de los cuentos incluidas en las categorías de Introduc- 
TABLA 2

Comparación de los sujetos con TEL y sujetos con desarrollo normal del lenguaje en medidas globales de producción de la narración

\begin{tabular}{lccccccc}
\hline \multicolumn{7}{c}{$\begin{array}{c}\text { Grupo Trastorno Específico de } \\
\text { Lenguaje }(\mathrm{N=25})\end{array}$} & \multicolumn{2}{c}{$\begin{array}{c}\text { Grupo de Comparación } \\
(\mathrm{N}=25)\end{array}$} \\
& $\begin{array}{c}\text { Media } \\
\text { (DE) }\end{array}$ & $\begin{array}{c}\text { Mediana } \\
\text { (IQR) }\end{array}$ & $\begin{array}{c}\text { Media } \\
(\mathrm{DE})\end{array}$ & $\begin{array}{c}\text { Mediana } \\
\text { (IQR) }\end{array}$ & $\begin{array}{c}\text { Estadístico } \\
\text { F/ U }\end{array}$ & $\begin{array}{c}\text { Signif. } \\
\text { Bilat. }\end{array}$ & $\begin{array}{c}d \text { de } \\
\text { Cohen }\end{array}$ \\
\hline Total Proposiciones & $36.67(16.97)$ & $38.89(27.78)$ & $67.33(7.74)$ & $66.67(8.33)$ & $U=34$ & 0.000 & -2.19 \\
Introducción & $45.33(30.24)$ & $33.33(33.33)$ & $72.00(24.87)$ & $66.67(33.33)$ & $U=166$ & 0.003 & -0.98 \\
Suceso & $38.50(20.38)$ & $37.50(25.00)$ & $68.50(9.62)$ & $75.00(12.50)$ & $U=73.50$ & 0.000 & -1.9 \\
Respuesta Interna & $20.00(40.82)$ & $0.00(0.00)$ & $84.00(37.41)$ & $100(0.00)$ & $U=112.50$ & 0.000 & -1.67 \\
Ejecución & $22.40(19.42)$ & - & $52.00(14.14)$ & - & $F=37.94$ & 0.000 & -1.78 \\
Consecuencia Directa & $32.00(47.60)$ & $0.00(100)$ & $72.00(45.82)$ & $100(100)$ & $U=187.50$ & 0.005 & -0.87 \\
Resolución & $52.00(50.9)$ & $100(100)$ & $100(0.00)$ & $100(100)$ & $U=162.50$ & 0.000 & -1.36 \\
Integridad de la historia & $26.27(13.87)$ & - & $88.56(10.01)$ & - & $F=331.14$ & 0.000 & -5.25 \\
\hline
\end{tabular}

Nota. Los datos se presentan como media/desviación típica, en el caso de seguir una distribución normal y/o como mediana/rango intercuartílico (IQR) en caso contrario.

Fuente: elaboración propia.

ción, $U_{(48)}=166, p<0.003, d=-0.98$, Suceso, $U_{(48)}=73.5, p<0.001, d=-1.9$, Respuesta Interna, $U_{(48)}=112.5, p<0.001, d=-1.67$, Ejecución, $F_{(1,48)}=37.94, p<0.001, d=-1.78$ y Consecuencia Directa, $U_{(48)}=187.5, p<0.005, d=-0.87$, a favor del grupo de comparación en todos los casos.

En cuanto a la nueva medida de integridad de la historia, es decir, la proporción de proposiciones fundamentales de la historia, el grupo TEL recuerda significativamente menos proposiciones fundamentales, $F_{(1,48)}=331.14, p<0.001, d=-5.25$. Tan amplías son las diferencias entre ambos grupos, que el grupo con TEL se encuentra a más de cinco desviaciones típicas por debajo del grupo de comparación.

\section{Resultados en medidas de errores de producción}

Los resultados que aparecen en la Tabla 3 señalan que se encuentran diferencias significativas, entre la ejecución de los dos grupos comparados, en ortografía natural, $U_{(48)}=107, p<0.001, d=1.43$ y en errores sintácticos, $U_{(48)}=131, p<0.001, d=1.17$, siendo el grupo con TEL el que comente mayor número de errores. Sin embargo, no se encuentran diferencias significativas en el número de errores de ortografía arbitraria, $F_{(1,48)}=2.64, p<0.111$, $d=0.46$, aunque el grupo con TEL comete mayor número de errores.

Los análisis de regresión múltiple (Tabla 4) pusieron de manifiesto que el modelo compuesto por tres variables (memoria secuencial auditiva, expresión verbal y comprensión auditiva) se mostró estadísticamente significativo, $F_{(3,46)}=29.157$; $p<0.001$, explicando el $63 \%$ de la varianza total de la variable integridad de la historia $\left(R^{2}\right.$ corregida $\left.=0.63\right)$.

\section{Discusión y conclusiones}

En relación con nuestro primer objetivo, comparar la sensibilidad las categorías estructurales de la historia de niños con y sin TEL, nuestros resultados señalan que los niños con TEL presentan un rendimiento en general más bajo que los niños normales en la tarea de recuerdo escrito de un cuento que previamente han oído. Este resultado está en consonancia con los hallazgos de los estudios que han empleado procedimientos distintos de evaluación del recuerdo de la narración. De hecho, los niños con TEL emplearon significativamente menos proposiciones a la hora de escribir sus historias, al igual que en las investigaciones previas que han valorado la narración de forma oral (p. e., Befi-Lopes et al., 2008; Botting, 2002; Epstein \& Phillips, 2009; 
TABLA 3

Comparación de los sujetos con TEL y sujetos con desarrollo normal del lenguaje en errores de producción de la narración

\begin{tabular}{|c|c|c|c|c|c|c|c|}
\hline & \multicolumn{2}{|c|}{$\begin{array}{l}\text { Grupo Trastorno Específico } \\
\text { de Lenguaje }(N=25)\end{array}$} & \multicolumn{2}{|c|}{$\begin{array}{c}\text { Grupo de Comparación } \\
(N=25)\end{array}$} & \multirow[b]{2}{*}{$\begin{array}{c}\text { Estadístico } \\
F / U\end{array}$} & \multirow[b]{2}{*}{$\begin{array}{l}\text { Signif. } \\
\text { Bilat. }\end{array}$} & \multirow[b]{2}{*}{$\begin{array}{c}d \text { de } \\
\text { Coher }\end{array}$} \\
\hline & $\begin{array}{l}\text { Media } \\
(D E)\end{array}$ & $\begin{array}{l}\text { Mediana } \\
\text { (IQR) }\end{array}$ & $\begin{array}{l}\text { Media } \\
(D E)\end{array}$ & $\begin{array}{l}\text { Mediana } \\
\text { (IQR) }\end{array}$ & & & \\
\hline Orto & $80(21$ & $3.00(4)$ & $360086-1-2$ & $0.00(0)$ & $\mathrm{U}=107.00$ & 0.000 & 1.43 \\
\hline Ortografía Arbitraria / & $8.56(3.44)$ & - & $7.08(2.98)$ & - & $F=2.64$ & 0.111 & 0.46 \\
\hline Errores Sintácticos & 2.36 (1.49) & $2.00(2)$ & $0.88(1.05)$ & $1.00(1)$ & $\mathrm{U}=131.00$ & 0.000 & 1.47 \\
\hline
\end{tabular}

Nota. Los datos se presentan como media/desviación típica, en el caso de seguir una distribución normal y/o como mediana/rango intercuartílico (IQR) en caso contrario.

Fuente: elaboración propia.

TABLA 4

Análisis de regresión múltiple: variables psicolingüísticas como predictoras de la integridad de la historia

\begin{tabular}{lcccc}
\hline \multicolumn{1}{c}{ Integridad de la historia } & $\mathrm{B}$ & $\boldsymbol{\beta}$ & $\boldsymbol{t}$ & $\boldsymbol{p}$ \\
\hline Memoria Secuencial Auditiva & 1.223 & 0.328 & 2.378 & 0.022 \\
Expresión Verbal & 1.866 & 0.281 & 2.622 & 0.012 \\
Comprensión Auditiva & 1.884 & 0.333 & 2.612 & 0.012 \\
$R=0.810, R^{2}=0.655$, Corregida $R^{2}=0.633$ & & & & \\
\hline
\end{tabular}

Fuente: elaboración propia.

Contreras \& Soriano, 2004; Manhardt \& Rescorla, 2002; Merrit \& Liles, 1987; Miranda, McCabe \& Bliss, 1998; Liles et al., 1995), aunque en diferentes trabajos se han observado diferencias de ejecución en niños con TEL en función de la modalidad de presentación de las historias (Schneider, 1996; Schneider \& Vis Dubé, 2005).

De acuerdo con los resultados del análisis más específico de las categorías de la gramática de la historia, los niños con TEL se diferenciaron de los niños del grupo de comparación en aspectos importantes relacionados con la manera como estructuraron sus narraciones. Así, al igual que ocurre en el recuerdo y producción de narraciones orales (p. e., Befi-Lopes et al., 2008; Botting, 2002; Copmann \& Griffith, 1994; Epstein \& Phillips, 2009; Liles et al., 1995; Manhardt \& Rescorla, 2002; Merrit \& Liles, 1987), los niños con TEL recuerdan por escrito significativamente menos información del cuento en las diferentes categorías del mismo Introducción, Suceso, Respuesta Interna, Ejecución,
Consecuencia Directa y Resolución. Esto es, omitieron más información sobre la presentación y los acontecimientos de la historia, las emociones del protagonista, así como de las acciones llevadas a cabo por los protagonistas para alcanzar la meta, así como de las consecuencias de dichas acciones y la resolución de la historia. Sin embargo, algunos estudios (Befi-Lopes et al., 2008) no encuentran diferencias en la categoría de respuesta interna ( $p$. e., captar las emociones del protagonista).

Además de escribir historias más cortas, los sujetos con TEL, en comparación con los niños con desarrollo normal del lenguaje, introducen menos proposiciones fundamentales en el recuerdo por escrito en la historia del relato. Este hallazgo sugiere que los niños con TEL tienen menos sensibilidad para discriminar la información relevante de la que no lo es. En otras palabras, en cuanto a esta última habilidad, estos chicos se sitúan a más de cinco desviaciones típicas con respecto al grupo de comparación, mientras que en relación con el 
total de proposiciones se sitúan a algo más de dos desviaciones típicas por debajo del grupo de comparación. Por desgracia, los problemas para captar las proposiciones fundamentales de la historia y, por tanto, las relaciones causales entre los eventos de la misma pueden tener importantes implicaciones para la vida diaria de los niños con TEL, no solo por la incidencia que tienen en la comprensión y composición de textos (Berthiaume, 2006), sino también porque podrían denotar dificultades en la comprensión social (Durkin \& Conti-Ramsden, 2010; Marton, Abramoff \& Rosenweig, 2005; Wadman, Durkin \& Conti-Ramsden, 2008).

En segundo lugar, con relación a la comparación de los niños con y sin TEL en cuanto a los errores de producción, nuestros resultados señalaron, en consonancia con otros estudios que emplearon muestras de escritura de niños y adolescentes con TEL (p. e., Bishop \& Clarkson, 2003; Dockrell et al., 2009; Dockrell et al., 2007; Mackie \& Dockrell, 2004), que los niños con TEL realizaron significativamente más errores de ortografía natural y sintácticos. Sin embargo, en contra de los hallazgos de estos estudios desarrollados con chicos de mayor edad, no se encontró que los niños con TEL cometieran más errores de ortografía arbitraria que los chicos del grupo de comparación.

Por último, en relación con el tercer objetivo, los resultados apuntan que los problemas lingüísticos de los niños con TEL pueden justificar sus dificultades en la narración escrita de historias. Esta vinculación causal ha sido evidenciada por otros estudios que han empleado tareas diferentes (p. e., Bishop \& Clarkson, 2003; Dockrell et al., 2007; Dockrell et al., 2009; Mackie \& Dockrell, 2004). Así, la comprensión auditiva, la memoria secuencial auditiva y la expresión verbal tienen gran importancia a la hora de predecir la habilidad narrativa de los niños con TEL. Sin embargo, en contra de algunos estudios (Mackie \& Dockrell, 2004; Scott \& Windsor, 2000), los datos del presente estudio señalan que la integración gramatical oral no tuvo ningún poder predictivo sobre la competencia narrativa en consonancia con algunos estudios (Bishop \& Clarkson, 2003; Dockrell et al., 2007), posiblemente por el escaso poder discriminativo de la medida de integración gramatical del ITPA.

Los resultados del presente trabajo contribuyen al progreso en el conocimiento de la competencia narrativa escrita de los niños con TEL, así como a la influencia de los déficits lingüísticos sobre dicha competencia. No obstante, habría que referirse a una serie de limitaciones que pueden afectar el alcance de los resultados. El tamaño de la muestra fue pequeño; en futuros estudios se deberán utilizar muestras más numerosas que aumenten el poder de los análisis estadísticos y hagan más factible la generalización de los resultados. Otra de las restricciones tiene que ver con la tarea de evaluación empleada; es posible que con otras tareas diferentes el perfil de resultados fuera diferente. Además, aunque las medidas lingüísticas empleadas están bien consideradas, se debería analizar en futuras investigaciones el rol de otras variables lingüísticas (p. e., Crespo \& Alfaro-Faccio, 2010; Gómez, Duarte, Merchán, Aguirre \& Pineda, 2007; Hincapié-Henao et al., 2008; Latorre \& EscobarMelo, 2010; Martínez, Bruna, Guzmán, Herrera, Valle \& Vásquez, 2001, 2002) y no lingüísticas (Hughes, Turkstra \& Wulfeck, 2009; Im-Bolter, Johnson \& Pascual-Leone, 2006; Marton, 2008) sobre la competencia narrativa.

Pero, con independencia de las limitaciones apuntadas, nuestros hallazgos tienen importantes repercusiones educativas, ya que la competencia narrativa constituye uno de los procesos organizativos fundamentales en los que se apoya el desarrollo representacional, la construcción de las bases del conocimiento y las estrategias de solución de problemas. Además, a medida que los estudiantes progresan en la escuela, las tareas escolares requieren la comprensión y producción de la estructura de las historias. Por ello, enseñar explícitamente los elementos de la gramática de la historia a los niños con TEL les ayudaría a superar sus dificultades con la escritura y con la comprensión lectora, como han mostrado diferentes trabajos, tanto en castellano (Acosta, Moreno, Axpe \& Lorenzo, 2010; Maggiolo, Pavez \& Coloma, 2003) como en inglés (Hayward \& Schneider, 2000; Stetter \& Tejero, 2010; Ukrainetz, 1998). 


\section{Referencias}

Acosta, V., Moreno, A., Axpe, A. \& Lorenzo, M. (2010). Apoyo al desarrollo de habilidades narrativas en niños con Trastorno Específico del Lenguaje dentro de contextos inclusivos. Revista de Logopedia, Foniatría y Audiología, 30(4), 196-205.

Aguado, G. (1999). Trastorno Específico del Lenguaje. Retraso de lenguaje y disfasia. Málaga: Aljibe.

American Psychiatric Association. (2000). Diagnostic and statistical manual of mental disorders (4a. ed. rev.). Washington, DC: Autor.

Befi-Lopes, D. M., Bento, A. C. \& Perissinoto, J. (2008). Narrations of stories by children with Specific Language Impairment. Pró-Fono Revista de Atualização Científica, 20(2), 93-98.

Berthiaume, K. S. (2006). Story comprehension and academia deficits in children with attention deficit hyperactivity disorder: What is the connection? School Psychology Review, 35(2), 309-323.

Bishop, D. \& Leonard, L. (2001). Speech and language impairment in children: Causes, characteristics, intervention and outcome. Oxford: Psychology Press.

Bishop, D., V. \& Clarkson, B. (2003). Written language as a window into residual language deficits: A study of children with persistent and residual speech and language impairments. Cortex, 39(2), 215-237.

Borzone, A. \& Diuk, B. (2003). La escritura de textos en niños pequeños: conocimiento ortográfico y producción textual. Cultura y Educación, 15, 7-27.

Botting, N. (2002). Narrative as a tool for the assessment of linguistic and pragmatics impairments. Child Language Teaching and Therapy, 18(1), 1-21.

Castro-Rebolledo, R., Giraldo-Prieto, M., HincapiéHenao, L., Lopera F. \& Pineda, D. A. (2004). Trastorno Específico del Desarrollo del Lenguaje: una aproximación teórica a su diagnóstico, etiología y manifestaciones clínicas. Revista de Neurología, 39(12), 1173-1181.

Cervera, C. \& Toro, J. (1984). TALE. Test de Análisis de la Lectoescritura. Madrid: Visor.

Chevrie-Muller, C. (1997). Trastornos específicos del desarrollo del lenguaje. En J. Carbona \& C. Chevrie-Muller (Eds.), El lenguaje del niño. Desarrollo normal, evaluación y trastornos (pp. 249-270). Barcelona: Masson.
Choudhury, N. \& Benasich, A. A. (2003). A family aggregation study: The influence of family history and other risk factors on language development. Journal of Speech, Language and Hearing Research, 46(2), 261-272.

Clegg, J., Hollis, C., Mawhood, L. \& Rutter, M. (2005). Developmental language disorders: A follow-up in later adult life. Cognitive, language, and psychosocial outcomes. Journal of Child Psychology and Psychiatry and Allied Disciplines, 46(2), 128-149.

Cohen, J. (1988). Statistical power analysis for the behavioural sciences (ed. rev.). New York: Academic Press.

Contreras, M. C. \& Soriano, M. (2004). El valor de la narrativa en la caracterización de alumnos con dificultades de lenguaje. Revista de Logopedia, Foniatría y Audiología, 24(3), 119-125.

Copmann, K. S. P. \& Griffith, P. L. (1994). Event and story structure recall by children with specific learning disabilities, language impairments, and normally achieving children. Journal of Psycholinguistic Research, 23(3), 231-248.

Crespo, N. \& Alfaro-Faccio, P. (2010). Desarrollo tardío del lenguaje: la conciencia metapragmática en la edad escolar. Universitas Psychologica, 9(1), 229-240.

Dockrell, J. E., Lindsay, G. \& Connelly, V. (2009). The impact of Specific Language Impairment on adolescent's written text. Exceptional Children, 75(4), 427-446.

Dockrell, J. E., Lindsay, G., Connelly, V. \& Mackie, C. (2007). Constraints in the production of written text in children with Specific Language Impairments. Exceptional Children, 73(2), 147-164.

Durkin, K. \& Conti-Ramsden, G. (2010). Young people with Specific Language Impairment: A review of social and emotional functioning in adolescence. Child Language Teaching and Therapy, 26(2), 105-121.

Epstein, S. -A. \& Phillips, J. (2009). Storytelling skills of children with Specific Language Impairment. Child Language Teaching and Therapy, 25(3), 285 300.

Gillam, R. \& Johnston, J. (1992). Spoken and written language relationships in language learning impaired and normally achieving school-age children. 
Journal of Speech and Hearing Research, 35(6), 1303-1315.

Gómez, L. A., Duarte, A. M., Merchán, V., Aguirre, D. C. \& Pineda, D. A. (2007). Conciencia fonológica y comportamiento verbal en niños con dificultades de aprendizaje. Universitas Psychologica, 6(3), 571-580.

Hayward, D. \& Schneider, P. (2000). Effectiveness of teaching story grammar knowledge to pre-school children with language impairment: An exploratory study. Child Language Teaching and Therapy, 16(3), 255-284.

Hincapié-Henao, L., Giraldo-Prieto, M., Lopera-Restrepo, F., Pineda-Salazar, D. A., Castro-Rebolledo, R., Lopera-Vásquez, J. P., et al. (2008). Trastorno Específico del Desarrollo del Lenguaje en una población infantil colombiana. Universitas Psychologica, 7(2), 557-569.

Howlin, P. \& Udwin, O. (2002). Outcomes in neurodevelopmental and genetic disorders. NY: Cambridge University Press.

Hughes, D. M., Turkstra, L. S. \& Wulfeck, B. B. (2009). Parent and self-ratings of executive function in adolescents with Specific Language Impairment. International Journal of Language and Communication Disorders, 44(6), 901-916.

Hulme, Ch. \& Snowling, M. J. (2009). Developmental disorders of language learning and cognition. Chichester, West Sussex, UK: Wiley-Blackwell.

Im-Bolter, N., Johnson, J. \& Pascual-Leone, J. (2006). Processing limitations in children with Specific Language Impairment: The role of executive function. Child Development, 77(6), 1822-1841.

Kirk, S. A., McCarthy, J. J. \& Kirk, W. D. (1986). Test Illinois de Habilidades Psicolingüísticas. Madrid: TEA.

Landis, J. R. \& Koch, G. G. (1977). The measurement of observer agreement for categorical data. Biometrics, 33(1), 159-174.

Latorre, D. C. \& Escobar-Melo, H. (2010). Tarea cognitiva de detección y corrección de errores en la comprensión de textos narrativos en niños de básica primaria. Universitas Psychologica, 9(3), 863-878.

Lê, K., Coelho, C., Mozeiko, J. \& Grafman, J. (2011). Measuring goodness of story narratives. Journal of Speech, Language, and Hearing Research, 54(1), 118-126.
Leinonen, E., Letts, C. \& Smith, B. (2000). Children's pragmatic communication difficulties. London: Whurr Publisher.

Liles, B., Duffy, R., Merrit, D. \& Purcell, S. (1995). Measurement of narrative discourse ability in children with language disorders. Journal of Speech and Hearing Research, 38(2), 868-882.

Mackie, C. \& Dockrell, J. E. (2004). The nature of written language deficits in children with SLI. Journal of Speech, Language and Hearing Research, 47(6), 1469-1483.

Maggiolo, M., Pavez, M. M. \& Coloma, C. J. (2003). Terapia para el desarrollo narrativo en niños con Trastorno Específico del Lenguaje. Revista de Logopedia, Foniatría y Audiología, 23(2), 98-108.

Manhardt, J. \& Rescorla, L. (2002). Oral narrative skills of late talkers at age 8 and 9. Applied Psycholinguistics, 23(1), 1-21.

Martínez, L., Bruna, A., Guzmán, M., Herrera, C., Valle, V. \& Vásquez, M. (2001). El efecto de recencia y el efecto de metría en la memoria de trabajo fonológica en niños con trastorno específico moderado del lenguaje. Revista Chilena de Fonoaudiología, 2(2), 19-30.

Martínez, L., Bruna, A., Guzmán, M., Herrera, C., Valle, V. \& Vásquez, M. (2002). Alteraciones en las representaciones fonológicas en la memoria de trabajo en niños preescolares con TEL. Revista de Logopedia, Foniatría y Audiología, 22(4), 181-189.

Marton, K. (2008). Visuo-spatial processing and executive functions in children with Specific Language Impairment. International Journal of Language and Communication Disorders, 43(2), 181-200.

Marton, K., Abramoff, B. \& Rosenzweig, S. (2005). Social cognition and language in children with Specific Language Impairment (SLI). Journal of Communication Disorders, 38(2), 143-162.

Mendoza, E. (2001). Trastorno Específico del Lenguaje (TEL). Madrid: Pirámide.

Merrit, D. D. \& Liles, B. Z. (1987). Story grammar ability in children with and without language disorder: Story generation, story retelling, and story comprehension. Journal of Speech and Hearing Research, 30(4), 539-552.

Miranda, A. E., McCabe, A. \& Bliss, L. S. (1998). Jumping around and leaving things out: A profile of 
the narrative abilities of children with Specific Language Impairment. Applied Psycholinguistics, 19(4), 647-667.

Monfort, M. \& Juárez, A. (1993). Los niños disfásicos. Madrid: CEPE.

Montgomery, J. W. (2003). Working memory and comprehension in children with Specific Language Impairment: What we know so far. Journal of Communication Disorders, 36(3), 221-231.

Norbury, C. F., Bishop, D. V. M. \& Briscoe, J. (2001). Production of English finite verb morphology: A comparison of mild-moderate hearing impairments and specific language impairment. Journal of Speech, Language, and Hearing Research, 44(1), 165-178.

Raven, J. (1995). Test de Matrices Progresivas de Raven. Madrid: TEA.

Restrepo, M. A. \& Gutiérrez-Clellen, C. (2001). Article use in Spanish-speaking children with Specific Language Impairment. Journal of Child Language, 28(2), 433-452.

Sanz, M. (2002). Los verbos en niños con trastorno del lenguaje. Revista de Logopedia, Foniatría y Audiología, 22(2), 100-110.

Schneider, P. (1996). Effects of pictures versus orally presented stories on story retellings by children with language impairment. American Journal of Speech-Language Pathology, 5(1), 86-96.

Schneider, P. \& Vis Dubé, R. (2005). Story presentation effects on children's retell content. American Journal of Speech-Language Pathology, 14(1), 52-60.
Scott, C. M. \& Windsor, J. (2000). General language performance measures in spoken and written narrative and expository discourse of school-age children with language learning disabilities. Journal of Speech, Language, and Hearing Research, 43(2), 324-339.

Stark, R E. \& Tallal, P. (1981). Selection of children with specific language deficits. Journal of Speech and Hearing Disorders, 46(2), 114-22.

Stein, N. L. \& Glenn, C. G. (1979). An analysis of story comprehension in elementary school children. En R. D. Freedle (Ed.), Advances in discourse processes. New directions in discourse processing (Vol. 2, pp. 53-119). Norwood, NJ: Ablex.

Stetter, M. E. \& Tejero, M. (2010). Using story grammar to assist students with learning disabilities and reading difficulties improve their comprehension. Education and Treatment of Children, 33(1), 115-151.

Tomblin, J. B., Records, N. L., Buckwalter, P., Zhang, X., Smith, E. \& O'Brien, M. (1997). Prevalence of Specific Language Impairment in kindergarten children. Journal of Speech, Language and Hearing Research, 40(6), 1245-1260.

Ukrainetz, T. A. (1998). Stickwriting stories. A quick and easy narrative representation strategy. Language, Speech, and Hearing Services in Schools, 29(4), 197-206.

Wadman, R., Durkin, K. \& Conti-Ramsden, G. (2008). Self-esteem, shyness and sociability in adolescents with Specific Language Impairment. Journal of Speech, Language and Hearing Research, 51(4), 938-952. 
